\title{
Frontières
}

\section{L'exil des ancêtres et le travail de deuil des adolescents antisociaux}

\section{Hélène de la Vaissière}

Volume 16, numéro 2, printemps 2004

Deuil, blessure vive

URI : https://id.erudit.org/iderudit/1074116ar

DOI : https://doi.org/10.7202/1074116ar

Aller au sommaire du numéro

Éditeur(s)

Université du Québec à Montréal

ISSN

1180-3479 (imprimé)

1916-0976 (numérique)

Découvrir la revue

Citer cet article

de la Vaissière, H. (2004). L'exil des ancêtres et le travail de deuil des adolescents antisociaux. Frontières, 16(2), 50-52.

https://doi.org/10.7202/1074116ar
Résumé de l'article

De nombreux jeunes incarcérés sont issus de familles émigrées. L’acte délictueux peut prendre le sens d'une représentation d'un deuil non réalisé à ou aux générations précédentes. L’acte délinquant s'adresse au champ social actuel et est une tentative d'inscription dans un environnement social et politique. 


\section{L'exil des ancêtres et le travail de deuil des adolescents antisociaux}

\section{Résumé}

De nombreux jeunes incarcérés sont issus de familles émigrées. L'acte délictueux peut prendre le sens d'une représentation d'un deuil non réalisé à ou aux générations précédentes. L'acte délinquant s'adresse au champ social actuel et est une tentative d'inscription dans un environnement social et politique.

Mots clés : adolescence - délinquance deuil-exil-intergénérationnel.

\section{Abstract \\ Many incarcerated youths are from immi- grant families. The infraction can acquire meaning as a representation of an unex- pressed grief of, or for, previous genera- tions. The delinquent act is towards the actual society as well as an attempt to find a space in a social and political environment.}

Key words: adolescence - delinquencygrieving - exile - intergenerational.

\author{
Hélène de la Vaissière, \\ psychologue clinicienne, Lyon, France.
}

La pratique clinique dans les prisons de Lyon confronte le psychologue à de nombreux jeunes adultes issus de l'immigration, plus particulièrement de l'émigration économique d'Afrique du Nord, en majorité algérienne et également à des jeunes issus des territoires d'outre-mer. À la migration des ancêtres (parents ou grands-parents) s'adjoint pour ces jeunes une similitude des zones d'habitat, limitrophes des grandes agglomérations.

Le regroupement de la population jeune délinquante sous l'égide des seuls critères psychopathologique (psychopathie) et sociologique (appartenance à des milieux sociaux défavorisés) laisse une faille dans la lecture clinique quotidienne. Aussi devons-nous chercher du côté de la particularité de l'histoire du sujet reliée à l'histoire familiale et communautaire, celle d'une émigration passée le plus souvent sous silence à l'intérieur des familles et dans la société.

La question identitaire est au centre des préoccupations de ces jeunes délinquants qui tendent à la résoudre : Comment faire vivre en soi la pluralité des différentes appartenances, familiales, sociales et culturelles passées et actuelles et en faire exister un métissage ? Les médias colportent l'image de jeunes pour lesquels les compromis sont de moins en moins possibles, image associée à une amplification de phénomènes de repli néo-identitaire (repli identitaire sur une pseudo-culture d'origine). Je fais l'hypothèse que l'acte délictueux prend sans doute en partie sens dans ces conflits identitaires et identificatoires actualisés dans le champ social et sans compromis intrapsychique.

Certains actes délinquants doivent être compris au sein d'une problématique transgénérationnelle de l'héritage de l'émi- gration / immigration, héritage d'une forme d'exil. La particularité de l'exil économique des populations d'Afrique du Nord, le plus souvent d'origine rurale, réside dans l'éventualité d'un retour. Cet éventuel retour entrave tout travail de séparation et d'individuation des migrants et de leur famille. Le travail de deuil nécessaire à l'intégration dans une nouvelle communauté et dans un nouvel espace politique reste en suspens pour la génération migrante et pour les générations descendantes.

Je souhaite faire part ici de quelques réflexions autour de la délinquance des jeunes issus de l'émigration / immigration et de leurs relations avec des deuils non effectués. Pour ce faire, j'aborderai les trois points suivants qui s'articulent entre eux: l'adolescence et ses deuils, l'exil des parents et la transmission d'un deuil non réalisé et une préhistoire inaccessible.

\section{L'ADOLESCENCE ET SES DEUILS}

La puberté impose un travail psychique de séparation et de deuil: "deuil de son corps infantile, du mode de penser infantile, de l'idéalisation attribuée aux parents" (Eiguer, 2001). Le travail psychique de détachement des représentants parentaux à accomplir au cours de l'adolescence passe par l'appropriation des objets transmis entre générations. Ladolescent interroge sa généalogie, les secrets familiaux, les pactes et les contrats dont il est l'un des protagonistes et dans lesquels il a été pris enfant. Il se pense à l'intérieur d'une filiation, d'abord dans sa position d'héritier puis lors du passage à une position adulte d'ascendant, créant sa propre famille, "chaînon de la chaîne des générations », selon l'expression de Freud.

Les agirs antisociaux sont à considérer comme des symptômes des difficultés à se penser, à entamer le travail de deuil des 
objets infantiles, à se confronter aux remaniements psychiques nécessaires à l'acquisition d'une position subjective et révèlent, outre les troubles actuels dans le processus de symbolisation, des failles des soutènements de la psyché par défaut de transmission à l'intérieur de la famille.

Ce défaut de transmission porte en partie sur l'impensable d'un traumatisme. «Les secteurs où leur pensée s'arrête correspondent bel et bien à des zones problématiques pour leur famille, et au premier chef pour leur parent», écrit Bernard Penot (2000). Les défenses narcissiques de ces adolescents visent à colmater les vécus traumatiques de la communauté. L'auteur parle de communauté de déni, nous pourrions aussi utiliser le terme introduit par René Kaës (1993) de pacte dénégatif. Le pacte dénégatif

\section{[...] s'impose dans tout lien}

intersubjectif pour être voué chez

chaque sujet du lien aux destins du refoulement ou de la dénégation, du déni, du désaveu, du rejet ou de l'enkystement dans l'espace interne du sujet ou de plusieurs sujets... chaque ensemble s'organise aussi négativement sur une communauté de renoncements et de sacrifices, sur des effacements, sur des rejets et des refoulements, sur un "laissés de côté » et sur des restes. La pacte dénégatif crée des zones de silence, des poches d'intoxication, des espaces-poubelles ou des lignes de fuite qui maintiennent le sujet étranger à sa propre histoire (Kaës, 1993, p. 274).

L'adolescent héritier de l'exil se trouve dans un environnement pénalisant le travail de deuil propre à cette période par le manque de transmission, les effets des secrets et le non-accès à sa propre histoire infantile et à sa préhistoire.

Le contexte historique des émigrations explique en partie ce phénomène. L'exil économique s'opérant le plus souvent sur une idéalisation du pays d'immigration suivie d'un silence sur les souffrances vécues perpétuant ainsi l'illusion des nouveaux candidats à l'émigration. Non seulement la famille mais l'ensemble du groupe social participe à cette communauté de déni. A contrario, l'agir délinquant de l'adolescent et du jeune adulte s'inscrit dans un mouvement psychique d'historisation nécessaire au processus adolescent, une tentative de symbolisation. Son sens est d'autant plus prégnant que l'entourage familial et social n'offre pas d'étayages suffisants à l'historisation et à la symbolisation. Le deuil des objets infantiles n'est pas exempt d'un autre deuil, celui qui n'a pas été psychiquement représenté.

\section{L'EXIL DES PARENTS ET LA TRANSMISSION D'UN DEUIL NON RÉALISÉ}

Ici, nous entendons l'exil au sens d'émigration / immigration d'un éloignement $d u$ pays d'origine :

[...] toute émigration est rupture, rupture avec un territoire et par là même avec une population, un ordre social, un ordre économique, un ordre politique, un ordre culturel et moral. Mais cause de rupture, l'émigration est elle-même le produit d'une rupture fondamentale : il faut que s'effondrent tous les cadres qui assuraient la cohésion de la société, pour que l'émigration puisse apparaître et se perpétuer (Sayad, 1999, p. 135).

L'exil garde une connotation politique d'appartenance à une communauté dont l'exilé est absent et d'un non-accès à une position de citoyen dans le pays d'accueil.

L'exil, quelle qu'en soit la cause, économique ou politique, contraint la psyché à un travail de deuil, deuil du pays d'origine. Or, comme nous le montrerons dans la vignette clinique, dans la population que nous avons rencontrée, le travail de deuil n'a pas pu se réaliser dans les générations précédentes, laissant le soin aux générations descendantes de l'entamer.

Dans son article Partages d'exil: la schizophrénie et l'objet politique interne, Jean Ménéchal (1997) émet l'hypothèse du politique tenant une place dans l'intrapsychique. Le politique serait alors un « objet secondaire de la structuration de la psyché et condition de celle-ci, à la fois construit par le social et organisateur du social conçu comme mode d'être collectif » (Ménéchal, 1997, p. 127). Le politique renvoie à l'espace public. J. Ménéchal pense un « objet politique interne » à partir des situations d'exil, «[...] l'exil, dans la complexité de ses partages, met au travail le champ même de la psychopathologie dans ses rapports au politique et constitue un des indices de cette dimension de la psyché» (1997, p. 28). L'objet politique interne « contient le répertoire potentiel des conduites de représentation non identificatoires du sujet au groupe ». Les enfants de l'exil sont « héritiers de l'exil d'un autre» et par conséquent « héritiers d'un exil de sa propre histoire». J. Ménéchal parle de nouage entre exil hérité et psychose, la psychose devenant "une solution à la situation d'héritage d'exil ». Les adolescents antisociaux ne se tournent pas du côté de la psychose mais vers des comportements délictueux, porteurs d'espoir, pour reprendre les termes de Winnicott. «La caractéristique essentielle de l'enfant antisocial est qu'il incommode, et c'est aussi, au mieux, un trait favorable indiquant à nouveau une nouvelle potentialité de recouvrer la fusion des pulsions libidinales et de motricité qu'il a perdue » (1956, p. 180).

Jean-Jacques Rassial soutient que l'acte s'adresse au champ social parce que celui-ci est ordonné par un discours qui tient, qui met de l'ordre en produisant du sens. David le Breton ajoute que les conduites à risques sont des tentatives "sauvages de symboliser sa place au sein du collectif, de se remettre au monde » (1998, p. 12).

L'acte délinquant en s'adressant au socius tente un compromis entre deux mondes clivés, celui d'un rattachement à deux communautés, et en particulier adresse le symptôme à la communauté d'accueil. Pour Sayad, l'exil est une double absence, absence à l'ailleurs et absence à l'ici. L'acte remet de l'ici et maintenant là où le temps s'est arrêté au seuil de l'adolescence, mais aussi là où il s'est arrêté pour les ancêtres émigrants.

La délinquance des jeunes issus de l'émigration / immigration prend un sens particulier lorsque l'on connaît le silence qui entoure l'émigration de leurs ancêtres et ses

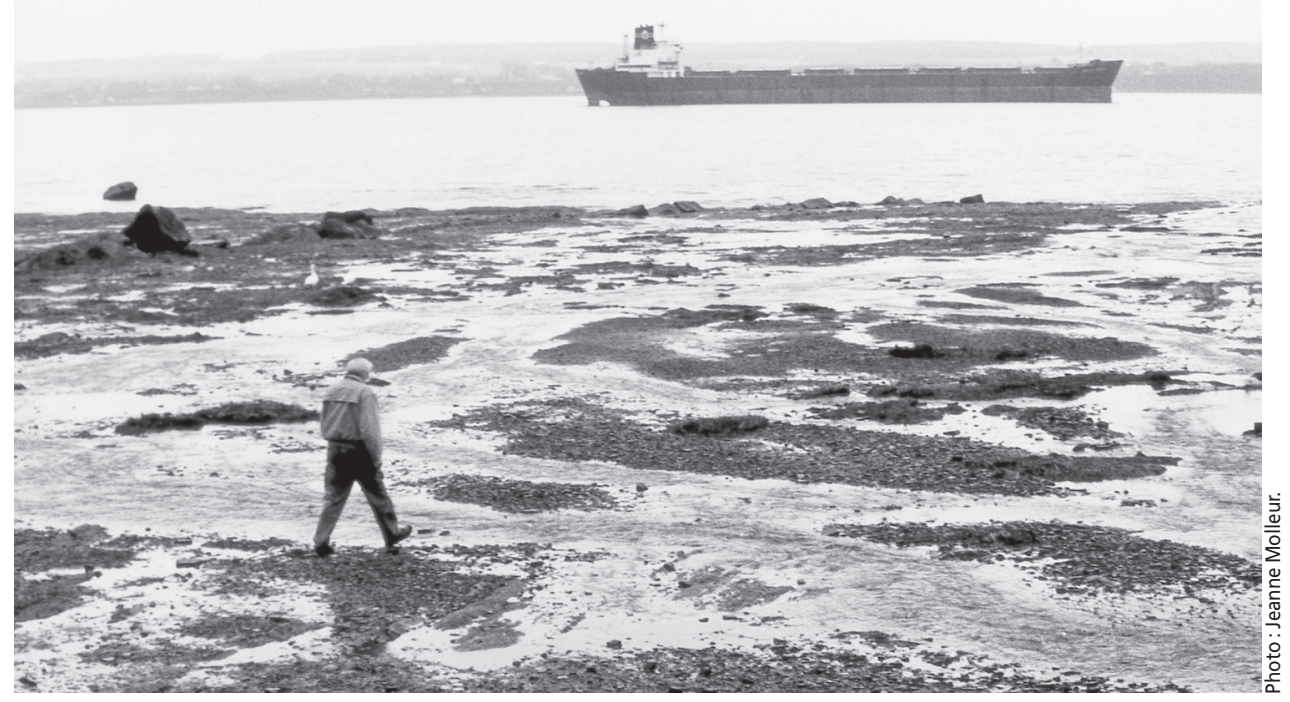


deuils. Elle est tentative d'inscription dans un environnement social et politique.

\section{UNE PRÉHISTOIRE INACCESSIBLE}

L'importance de la transmission intergénérationnelle n'est plus à démontrer. Elle offre au sujet un terreau préhistorique sur lequel il va fonder sa propre conception de ses origines, de sa généalogie et construire son identité.

Les traumas, les souffrances, non transmis, ceux de toute une communauté laissent un vide de pensée, un vide de construction d'une fiction de la préhistoire. À cet état de fait s'ajoute l'aspect anhistorique des quartiers d'accueil. Les banlieues construites pour accueillir des populations migrantes, des rapatriés, des réfugiés, des exilés demeurent sans histoire, construites et détruites au rythme des politiques de la Ville. Ces jeunes vivent dans des espaces non investis comme lieux de mémoire. L'urbanisation des banlieues ne favorise aucune mémoire culturelle, collective ou encore individuelle. Les adolescents n'y trouvent aucun support à une activité fictionnelle nécessaire à la construction d'un Je. Aux repères spatiotemporels brouillés des enfants d'exilés (Ménéchal, 1997) se joint cet espace urbain atemporel. Les zones d'habitat qui assurent difficilement leur fonction de mémoire renforcent et redoublent les difficultés d'appropriation d'une histoire, d'histoires ${ }^{1}$.

\section{MOUNIR, L'IMPOSSIBLE ACCÈS AUX TRAUMATISMES}

Mounir a tout juste 18 ans et vient d'être condamné à six mois d'emprisonnement pour vol de voiture, " une belle voiture, un rêve de la conduire ». Il est condamné pour la première fois en France.

Mounir a accepté de participer aux entretiens de recherche menés en vue de ma thèse. Je l'ai rencontré à quatre reprises. Cependant il n'a de cesse de demander à être rapproché d'un cousin incarcéré dans un autre quartier pénitentiaire. Il souhaite être protégé, ne sort plus de cellule, craint des rencontres. Sa demande aboutira après le second entretien.

Mounir aborde avec moult précautions l'histoire d'une première incarcération en Tunisie. Alors qu'il y passait des vacances l'année de ses 15 ans, il participe à une bagarre entre deux bandes rivales au cours de laquelle un jeune homme trouve la mort.

Mounir est né d'un couple mixte de père algérien et de mère française. Ses parents se sont séparés lorsqu'il avait 15 ans et son père est rentré dans son pays d'origine. Il ne donne que rarement signe de vie. La mère s'occupe de ses trois enfants dont Mounir est le cadet. Mounir a suivi ses études jusqu'en $3^{\mathrm{e}}$ puis a été contraint à changer d'établissement à cause de pro- blèmes de discipline. Mais il n'a pu se soustraire à fréquenter un collège hors de son quartier et très vite il a cumulé les absences.

Mounir relate peu de chose de sa vie familiale ; il dit ne rien connaître des migrations de sa mère (changement de région) et de son père. En effet, la mère de Mounir a quitté le Nord de la France, a vécu quelque temps à Paris et s'est installée dans la région lyonnaise où elle a rencontré le père de ses enfants. Mounir est surpris par ma question sur l'histoire de l'émigration de son père. Cependant, il est en capacité de noter des noms de sa famille élargie maternelle et paternelle sur un arbre généalogique. L’ordre générationnel n'y est pas respecté. Il situe sa fratrie entre ses parents et les familles respectives de l'un et de l'autre. La situation actuelle de son père reste vague... entre France et Algérie dans une impossibilité à le situer géographiquement.

Mounir témoigne peu de l'histoire de sa propre enfance, est absent de l'histoire de ses parents aussi bien du côté maternel que paternel et se trouve dans l'impossibilité de quitter les lieux de proximité de son habitation. Les migrations ne sont pas expliquées, ne donnent lieu à aucun mythe. Nous retenons d'un côté la peur, peur de l'autre vécu comme dangereux, dans un danger imminent qui demande de s'en éloigner rapidement dans une relation quasi de vie et de mort.

Le cas de Mounir est paradigmatique des situations que nous rencontrons en milieu carcéral et qui entrave toute mise au travail psychique. Ces jeunes non seulement n'ont pas de repères de leur propre histoire, mais encore moins de l'histoire des générations qui les ont précédés.

Le meurtre en terre étrangère correspondrait-il au meurtre d'une part de l'histoire du père en arrivant sur la terre de France?

La paradoxalité spatiale (Ménéchal, 1997) est de trois sortes : séparer sans transformer, s'éloigner en restant proche, se perdre en restant au même endroit... L'héritier de l'exilé sent la présence de l'ailleurs que les siens ont fui. Le changement suppose une réappropriation de l'initiative de déplacement. La demande de changement de quartier pénitentiaire sous-entend un rapprochement vers le connu, un besoin de protection, une pulsion d'autoconservation.

L'espace clos de la voiture volée, les difficultés de s'éloigner du quartier lors du changement d'établissement scolaire, son souhait de se rapprocher du quartier pénitentiaire où séjourne son cousin ramène à l'impossible éloignement, à l'incapacité de penser une séparation au sein d'une histoire familiale habitée par une double émigration. Lincarcération fait fonction de pseudo-séparation tout en s'inscrivant dans les lois et les règles du champ social auquel elle appartient.
Tout semble ramener à la clôture, à une impossible séparation. L'absence d'histoire de l'exil de ses deux parents laisse une psyché en exil d'elle-même, exilée de sa propre préhistoire. Mounir est confronté à de l'impossible à penser qui ne peut le laisser qu'en errance et en déshérence. A contrario, l'acte délinquant l'inscrit socialement.

En prison, les mouvements psychiques de désespoir et de revendication raniment le sujet sans assises historiques. Ils s'apparentent aux mouvements psychiques propres au deuil.

L'adolescence contraint à une mise en fiction des origines. L'émigration/immigration parentale appartient à la fois au non-dit des familles et au silence social. Les adolescents issus de parents ou de grands-parents émigrés restent avec un vide de préhistoire qu'ils tentent de résoudre à travers un acte porteur d'espoir. L'acte délinquant permet alors de s'inscrire dans une société et de symboliser l'absence.

\section{Bibliographie}

EIGUER, Alberto (2001). La famille de l'adolescent, le retour des ancêtres, Paris, Press Editions.

KAËS, René (1993). Le groupe et le sujet du groupe, Paris, Dunod.

LE BRETON, David (1998). Violences, conduites à risques et jeunes générations, Actes du colloque de Tours, p. 11-23.

MÉNÉCHAL, Jean (1997). Partage d'exil : la schizophrénie et l'objet politique interne, Cliniques méditerranéennes, $\mathrm{n}^{\circ} 62$.

PENOT, Bernard (2000). La passion du sujet freudien. Entre pulsionnalité et signifiance. Ramonville, Érès.

Latence, sublimation, adolescence, SPP en ligne, 2000.

RASSIAL, Jean-Jacques (1998). Y a-t-il une psychopathologie des banlieues?, Ramonville, Érès.

SAYAD, Abdelmalek (1999). La double absence, Paris, Seuil.

De la VAISSIÈRE, Hélène (2003). Clinique du lien social des jeunes délinquants. Relations au groupe, au socius et aux institutions de jeunes incarcérés aux prisons de Lyon, Thèse présentée à l'Université Lyon II.

WINNICOTT, D.W. (1969 [1956]). «La tendance antisociale », dans De la pédiatrie à la psychanalyse, Paris, Payot.

\section{Note}

1. Depuis peu, l'histoire des banlieues fait l'objet de recherches auprès des habitants. Dans le cadre de la biennale d'art contemporain en 2003, le quartier de la Duchère a exposé dans un bus des interviews d'habitants du quartier, tous migrants, réfugiés politiques ou rapatriés, récents ou plus anciens. Une ethnologue, Louisa Charfa, a réalisé trois films sur le quartier du Tonkin à Villeurbanne sur le thème «Quartier et origine ». 